\title{
Social Work and Youth Sport
}

\author{
Dawn Anderson-Butcher ${ }^{1} \cdot$ Samantha Bates $^{1}$
}

Accepted: 24 May 2021 / Published online: 28 May 2021

(c) The Author(s), under exclusive licence to Springer Science+Business Media, LLC, part of Springer Nature 2021

For decades, social work has leveraged sport as a context for prevention, intervention, research, and advocacy. In fact, scholars argue Jane Addams, a prominent social work leader, used sports and athletics to promote health and well-being, foster community and acculturation, build relationships among people and institutions, and engage hard-to-reach immigrant populations in the early 1900s (Lawson \& Anderson-Butcher, 2000; Reynolds, 2017). In the last decade, sport social work has emerged as one of the most exciting new areas of social work practice, education, and research (Moore \& Gummelt, 2018). The Alliance for Social Workers in Sport was formed in 2015 to represent the profession's voice in sport contexts, as well as promote, encourage, and support the growth of the subdiscipline of sport social work.

Growth of sport social work is evident across multiple settings, including professional and collegiate sports organizations, as well as in youth sport contexts. Specific to youth, social workers leverage sports in afterschool, school, and community-based settings as they address imperative mental and health challenges; promote and renew health and wellness in the lives of individuals, families, groups, and communities; and engage multiple sectors in sports' traditions, events, and rituals in support of belonging, connectivity, and social capital. Today, social workers and other professionals implement sport-based interventions in settlement houses, correctional facilities, schools, community centers, behavioral mental health agencies, parks and recreational facilities, and youth development settings such as at the Boys \& Girls Clubs and YMCA. Furthermore, approximately 50\% of children and adolescents worldwide participate in organized sports, where youth learn life skills such as teamwork and leadership, widening the reach of sports for social development (Anderson-Butcher, 2019; The Aspen Institute Project Play, 2019). Youth sport, in particular, also engages parents/

Dawn Anderson-Butcher

anderson-butcher.1@osu.edu

1 College of Social Work, The Ohio State University, 1947 N

College Rd College of Social Work Columbus, Columbus,

OH 43210, USA caregivers, families, siblings, other caring adults, coaches, fans, and sports administrators in supporting positive youth development (PYD).

Given its universal appeal and mass participation rates, youth sport has the potential to influence health and mental health outcomes, reduce problem behaviors, foster social inclusion, and promote broader social welfare. Indeed, evidence suggests youth sport participation predicts a host of positive youth outcomes, especially for youth from socially vulnerable circumstances (Anderson-Butcher, 2019; Eime et al., 2013; Spruit et al., 2016; Whitley et al., 2019). Social work interventions using sport, however, have been understudied, especially the ones targeting youth from diverse backgrounds (Anderson-Butcher et al., 2013). As a result, social work scholars have opportunities to address disparities in access and participation; elevate the voices of vulnerable youth and their families; address issues related to discrimination, prejudice, and bias; identify culturally competent coaching and parenting supports; and ensure ethical practices and equity in sports.

Additionally, leveraging social work and sport aligns with our Social Work Grand Challenge to ensure the healthy development of all youth (Jensen \& Hawkins, 2018). The impetus for this special issue pre-dated the COVID-19 pandemic and the Black Lives Matter movement. However, the nine papers included in this special issue highlight examples of how youth sport buffers risk and increases protective factors across multiple systems. More specifically, this special issue collates the work being performed by social workers and other scholars in youth sport settings, to advance our interprofessional collaboration and leadership in the field. In addition, researchers contributed articles that allowed us to explore applications of social work practice in sport across the micro-macro continuum. As guest editors, we have organized this special issue around two broad domains: (a) prevention and intervention leveraging youth sports and (b) implementation, policy, and advocacy. 


\section{Prevention and Intervention in Youth Sport}

An exciting group of articles highlights the important roles that social workers and others are taking when creating prevention, PYD, and social work interventions in youth sport Notably, researchers contributed articles that overview innovative and creative approaches to researching prevention and intervention strategies embedded in sports, which seek to enhance these and other protective factors for youth. In addition, authors' contributions built upon past work to ensure sport programming for socially vulnerable populations is high quality, evidence-based, and grounded in theory. Certainly the innate features of any sport require skills such as risk taking, social interactions, problem solving, emotional regulation, leadership, and communication. Outcomes such as these can be amplified through intentional practices targeting specific groups of young people.

One gap in the sport-based PYD literature focused on what design elements contribute to successful life skill development and broader physical and mental health outcomes for youth. Two articles in this special issue examined important design elements that support positive social and health outcomes for youth attending sports-based PYD summer camps. Anderson-Butcher and colleagues explored whether social-emotional learning (SEL) curricula and instruction delivered via verbal reinforcements and tangible incentives (i.e. buttons) positively influenced the development of social skills among socially vulnerable youth. Implications of this article contributed to a greater understanding of evidence-based design and teaching mechanisms in youth sport contexts that facilitate social skill development, including the use of positive behavioral supports and peer supports. An article by Henert and colleagues examined the effect of participation in a six-week physical activity-based summer day camp. Camp PAL is grounded in the Teaching Personal \& Social Responsibility model (TPSR) and aims to teach urban youth life skills through sports and games. Results suggested sport-based summer PYD camps may help diverse populations maintain adequate daily physical activity levels while simultaneously promoting their broader social welfare.

Another important topic central to the development of effective youth sport interventions is the training of program leaders and youth sport coaches. Jacobs and Wahl-Alexander examined four graduate students' selfefficacy beliefs to lead a leadership and life skills sports program in a youth prison. Researchers offered insights into how individuals perceive their competencies for leading a sport program within a highly volatile social welfare environment. Their study found that graduate students' self-efficacy beliefs about their abilities to be successful was related to their level of preparation and verbal encouragement received from faculty supervisors and facility partners. These and other findings have important implications for training professionals to use sports in practice, in typical social work practice settings which are non-traditional sports.

Additionally, Moore and colleagues explored the implementation of the five Cs model (competence, confidence, connection, character, and caring/compassion; Lerner et al., 2005) in a youth academy soccer program. Using a mixed methods longitudinal approach, scholars found the implementation of the five Cs was associated with more opportunities for positive development among youth athletes. They specifically found increased positive affect among youth athletes, an increase in parental support of the academy, and a shift in coaching philosophies, towards the promotion of five Cs. Together, these studies advance scholarship in the areas of program design, life skill development through youth sport, and best practices in coaching, mentoring, and sport-based PYD programming.

Two additional studies focus on how sport-based PYD programs contribute to life skill transfer, specifically exploring how youth use the various skills learned in sport in other areas of life. Newman and colleagues explored eight psychological processes contributing to the transfer of life skills learned in sport. Specifically, they used photo-elicitation methods to empower youth, allowing them to share their unique lived experiences post-participation in a sport-based PYD program. This study contributes to methodological advancements in the field through the use of elicitation methods, to elevate the voices of youth participants. A second study examined access and life skill transfer via sport-based PYD delivered virtually during the COVID-19 pandemic. Specifically, Bates, Greene, and O'Quinn utilized a mixed method approach to explore the accessibility and the lived experiences of youth and families who participated in a virtual sport-based PYD programming. This article provided a unique opportunity to understand access and engagement in virtual sport activities during the lockdown due to the pandemic. Findings indicated high participation rates in virtual sport-based PYD activities and found virtual activities facilitated life skill transfer. Collectively, these articles point to the importance of taking what one learns in sport and using those skills in other social settings. Life skill transfer is an important developmental mechanism for learning, and is especially important given decreased opportunities for sports, PYD, and social interaction during the COVID-19 pandemic.

\section{Implementation, Policy, and Advocacy}

The second set of articles in this issue explore broader organizational, familial, and interpersonal factors in sports. As a profession, we have long embraced an ecological 
perspective in facilitating change across systems. Social workers see beyond direct service situations and work to enhance organizational change efforts, policy practice, and community youth development. Several articles in this special issue highlight the unique ways trauma-informed care, policy practice, and advocacy are shaping various sport and social work settings. One such example by Shaikh and colleagues described the integration of sport-based traumasensitive practices with the Boys \& Girls Club of Canada's national Bounce Back League program. Authors used a mixed methods approach to generate insights into staff training experiences, successes and challenges of program implementation, and the potential impact of the program on club members. Lessons learned from this important work help us understand further how sport settings can develop, adapt, and integrate trauma-sensitive practices in community contexts.

Coming from a macro-policy approach, the Reynolds article sought to understand how the passage of Act 355 in the 2019 Louisiana Legislature influenced spectator behaviors, including those of parents. This policy in Louisiana applied to all sanctioned events (competitive and recreational) in the state and outlined consequences for demonstrating verbal and/or physical abuse, both on and off the field. The law did not prohibit disagreements with officials but disallowed verbal and non-verbal behaviors that could place a person at risk of harm or injury. Reynold's work is novel; few studies engage in policy practice by exploring outcomes with interventions designed to educate youth sport parents.

Arinze and McGarry's contribution is a call to advocacy. These scholars described the experiences of Black and Latina girls in sports as a way to argue for more sport-based PYD research, using intersectional approaches. In their article, they contend that challenging traditional narratives and elevating the lived experiences of Black and Indigenous girls of color provide platforms for adolescents to contribute towards changes in sport design strategies that honor youth voices, validate young people's wisdom, and spark transformation toward equity. Articles present the various ways social workers in youth sport contexts can address complex social challenges across multi-dimensional systems.

\section{The Future of Social Work and Youth Sport}

This special issue provides a foundation for elevating and advancing the subdiscipline of sport social work by emphasizing the growing body of research on social work and youth sport. Leaders in the field can build off these findings to support social workers and other professionals as they spark social change and leverage sports to improve the health and well-being of children, adolescents, families, and communities. Authors envision opportunities for researchers, educators, practitioners, policymakers, and youth sport participants to work collectively to advance our understanding of the value of this important practice domain. As we strategize for the future, we share a myriad of opportunities to contribute to this growing area of social work practice, education, and research. One way forward is to think about ways to leverage sport for social change, using our knowledge and practice across the micro-macro continuum, our professional mission, and our commitment to the Social Work Grand Challenges (Jensen \& Hawkins, 2018). Next, we detail five important areas for the future development and growth of scholarship and practice on social work and youth sport.

\section{Enhance Knowledge of How Youth Sports Support Individual, Family, Community, and Global Development}

Social workers recognize sport has the potential to impact individuals, teams, families, and communities. As a result, there are a myriad of opportunities for scholars to leverage the systems perspective to explore the value of youth sport from a social work lens. Although often a secondary outcome associated with participation, sports are important settings for addressing risk factors and promoting assets evident in families, schools, peer groups, and communities. In fact, there is some evidence to suggest youth sport may promote family involvement and communication, school climate and engagement, peer relationships and attachment, and broader social development (Anderson-Butcher, 2019; Lower-Hoppe et al., in press; McDonough et al., 2017).

As scholars, we argue sports' innate convening power holds promise in strengthening prevention efforts that contribute to broader systemic changes in communities and globally. However, more research on the role of sports in prevention is needed to develop links among youth sport participation, positive youth developmental outcomes, and reduction in community- and global-level risk factors (such as gang involvement, school dropout, teen pregnancy, obesity, social exclusion, and violence). Scholarship on innovative youth sport design strategies, community and global investments in sports, and promising national or international models that use sport as a tool for prevention can advance the field and, most importantly, contribute to structural change strategies. In fact, the newly adopted National Youth Sport Strategy (NYSS; U.S. Department of Health \& Social Services [DHHS], 2019) calls for all youth to have the access and opportunity regardless of circumstances because of the public health value of sports for the health of young people and communities. The policy context is set for the promotion of healthy developmental outcomes through sport. 
Investments in youth through sports are thought to have the potential to support the development of healthy adults to cultivate a more prepared and civically engaged workforce (Anderson-Butcher, 2019). At the structural level, sports can also improve social outcomes including building community, social capital, and social cohesion; promoting educational attainment and employability; addressing food insecurity; and improving mental health, well-being, and longevity (Karakaya, 2014). By furthering our knowledge on the ways in which sports promote community and global development, we can not only learn more about how sport can address the broader social problems youth face.

\section{Investigate Best Practices in Youth Sports}

Offering youth sport alone is not enough to improve conditions for youth and families. As a profession that values human relationships, competence, and integrity, social workers have a unique opportunity to advance our understanding of effective ways, to maximize the experiences of youth in sport and empower our next generation of leaders. Maximizing sport as a developmental context is especially important, given $50 \%$ of young people participate in organized sports across the world (Hulteen et al., 2017). Social work scholars and practitioners can advance best practices in youth sport by uncovering the ways nonacademic and prosocial skills (i.e. public speaking, self-efficacy, college and career readiness, decision-making) can be "taught and caught" through sports (Gould \& Carson, 2008; Pierce et al., 2016). By identifying evidence-based mechanisms that strengthen sport as a context for learning and belonging, social workers will have access to additional opportunities, to engage youth in activities focused on civic engagement, leadership, and advocacy.

Further, life skill development through traditional sport research is growing, yet more research is needed to better understand life skill transfer to other social settings. Scholars contend there are limited studies examining the value of sport for youth who are most socially vulnerable (Anderson-Butcher, 2019; Hermens et al., 2017), namely those the profession of social work is most committed to supporting. In addition, positive developmental outcomes associated with participation in sport are often reliant upon many factors within the developmental settings (i.e. funding, resources, program designs, curricula, coaches, equipment, facilities, and mechanisms associated with teaching and learning). In relation to sport settings specifically, there is a need to explore the characteristics of programs influencing process and outcomes, furthering our understanding of what works for different types of youth and under what conditions (Anderson-Butcher, 2019; Riley \& AndersonButcher, 2012). Attention to the impact of sports on youth from socially vulnerable circumstances, girls, youth of color,
LGBTQIA + youth, and those with disabilities is growing in priority for future research studies (DHSS, 2019). Advancing our understanding of the best practices that make youth sport settings safe and effective through rigorous research designs will only strengthen our opportunities to leverage sports for PYD, population health, and social change.

\section{Develop Translational Models for Social Work Practice in Clinical, School and Community Settings}

A key element of social work practice is drawing upon the evidence-base whereby research informs practice and practice informs research. As new knowledge of evidence-based practices related to social work and youth sport emerge, we must turn our focus to translational science and dissemination of best practices to clinical, school, and community settings. One in five youth experience some type of behavioral health problem in their lifetime, which in turn predicts a host of negative outcomes, such as school failure, violence, substance use, and other social problems (Domitrovich et al., 2017; Jenson \& Hawkins, 2018; O'Connell etal., 2009). Understanding how sport can be leveraged to support and buffer risks of behavioral health concerns remains paramount to informing future interventions and clinical practice models. Past research demonstrates that sport and physical activity, when designed with intentionality and quality, have the potential to significantly impact the mental health of young people (Eime et al., 2013).

Leveraging sports in treatment settings certainly has value for improving child and adolescent outcomes. Limited research, however, exists on exploring the value of sport in therapeutic or clinical settings. One wonders how evidencebased practices known to be effective sport research (such as goal setting, self-talk, imagery, relaxation, etc.) can be applied in clinical social work practice settings with children and adolescents. Ultimately, there is much to learn about how interventions in youth sport support the Social Work Grand Challenges efforts to address the healthy development of all youth, particularly in relation to behavioral health. We also have opportunities to distill what social work and sport practices work best for specific subgroups of youth, as one size does not fit all. Few studies to-date use experimental designs, and there is an overreliance on case studies and qualitative methodologies. (Coalter, 2010; Eimeet al., 2013; Hermens et al., 2017; Holt et al., 2017; Jones et al., 2016)

One promising area where there has been significant attention in social work practice relates to SEL, an area also emphasized in some of the papers in this special issue. A framework developed by CASEL (2017), which is arguably the most widely known and utilized in schools, identifies the five core domains of SEL: (a) self-awareness, (b) selfmanagement, (c) social awareness, (d) relationship skills, and (e) responsible decision-making. This framework is 
highly relevant for school and sport social workers, as SEL expands the focus of interventions to not just individuallevel skill-building but also to the context. SEL curricula and universal interventions in schools and sport have the potential to support teachers, social workers, and coaches in building healthy relationships and responding to youth needs. The Aspen Institute (2018) recently called upon youth sport coaches to integrate social, emotional, and cognitive skill building into their programs, to foster athletes' holistic development. As social workers, we recognize the utility of SEL as a tool for prevention that allows youth to develop skills before experiencing an adverse or stressful event.

SEL skill development learned in sport is an area of research and practice that social workers can explore more fully. In fact, participation in sport focused on SEL skill development may increase a myriad of protective factors such as helping youth identify their emotions and cope with stress (Anderson-Butcher, 2019). The long-term implications of learning SEL skills in youth sport may buffer future risks for depression and anxiety among children and adolescents. Further, intentionally developing SEL skills in sport has the potential to create high-quality sport environments, to cultivate a love of sport and collaboration among youth, and to prepare athletes to excel in sport, life, relationships, and beyond (The Aspen Institute, 2019). Researchers advocate gaps exist in the development and utilization of researchsupported measurement tools, to assess and inform SEL in sport contexts. We also have opportunities to translate skillbuilding activities that exist in sports to other practice settings, such as schools and community youth. By translating best practices in sport-based SEL skill development across the research and clinical practice continuum, social workers can play a huge role in leveraging youth sport to improve the lives of socially vulnerable children and adolescents.

\section{Strengthen Sport-based Social Work Training and Education Programs}

Sport social work has grown exponentially over the last decade. Developmentally, with the changing of the Education Policy and Accreditation Standards (EPAS) social work competencies in 2022, the timing is right for the social work profession to examine ways to strengthen workforce training and development programs focused on equity, anti-racist practice, and advocacy through sport. This includes preparing practitioners to engage in ethical and evidence-based practice within diverse sport contexts including youth sports or professional or collegiate sports teams. There are also opportunities for social work educators to envision ways for sports, recreation, and games to become threaded into the mainstream social work curriculum and tied to social work competencies, at both the undergraduate and graduate levels. Teaching courses about sport, recreation, and games go hand-in-hand with social work curricula and interventions focused on behavioral health, attachment, boundaries, rapport-building, program design, and policy practice.

The profession can work to enhance training opportunities by developing sport-based field placements, sport social work courses, interprofessional education activities grounded in sports (i.e. concussion management, teambased care for athletes), and continuing education units that enhance the skill sets of current or future social work practitioners. As of today, only a few sport social work courses exist across the country despite a growing number of job opportunities for social workers emerging in athletic departments and community sports settings. Based on a growing number of jobs posted by the Alliance for Social Workers in Sport, social workers are well-equipped to work with student-athletes, coaches, and athletics department staff, and to develop relationships with alumni, donors, campus partners, and employers for the purposes of better preparing studentathletes for meaningful careers beyond sport. Strengthening areas of education and training will only continue to grow the specialization of sport social work, and ultimately, benefit those who receive social work services.

\section{Utilize Sports to Advance Human Rights and Social Justice}

Social work's focus on assessing needs and empowering individuals who are vulnerable, oppressed, and living in poverty will bring a new lens to youth sport research. Based on articles included in this special issue, the social work lens can help better understand the intersectional and structural factors influencing sports participation, and how barriers to access and opportunities may be addressed in socially vulnerable communities. Additionally, social workers have the knowledge and skills to call out White supremacy/superiority (and its ensuing privilege) and to engage in anti-racist practice. As evidenced by the Black Lives Matter and MeToo Movements, social workers in sport contexts and elsewhere can join in the fight with sport leaders, teams, and organizations and leverage our platforms to elevate the needs and voices of the socially vulnerable while also demanding justice from oppressive institutions.

Within sport organizations and among athletes, we have witnessed a re-emergence of social justice advocacy and athletes using their capital to invest in the lives and well-being of historically marginalized and oppressed communities. Recent Nike commercials focused on social justice, equity, and equality, and the boycott of NBA games demonstrate how advocacy through sport has the potential to reach the masses and push forward social policy. The needle is also moving forward on topics such as gender equality and access to sport for girls. For instance, the Angel City Football Club is looking to make an impact on women's sports and soccer 
with new community initiatives as the team prepares to join the National Women's Soccer League in 2022. Furthermore, the engagement of athletes and sport organizations in socially just causes and advocacy campaigns have the power to transform society. A few examples of athletes and sport organizations investing in the development of youth include (a) LeBron James' I Promise School in Akron, Ohio; (b) Learning in Fitness and Education through Sports (LiFEsports) at The Ohio State University; and (c) the Los Angeles Football Club's Youth Leadership Program in Los Angeles, California. Social workers have immense opportunities to partner with sport leaders, teams, and organizations to empower youth and communities through sports and athletics.

As a profession, social workers value self-determination, cultural humility, and the lived experiences of our clients. What is often missing in our research, practice, and education on sport is the voice of the child, adolescent, family, and community. There is an imminent need for social work scholars and practitioners to elevate the stories, narratives, cultural capital, and experiences of clients, to ensure we implement culturally responsive, sustainable, and equitable interventions that promote human dignity for all. Furthermore, we need scholars that interrogate harmful practices within sport contexts themselves, to ensure programs, coaches, and peers do not contribute to structural oppression, marginalization, or trauma for youth participants. Finally, policy and advocacy work in sport can move towards increasing accessibility, equity, and quality in the youth sport delivery system, especially in socially vulnerable groups (i.e. age, class, color, culture, disability and ability, ethnicity, gender, gender identity and expression, generational status, immigration status, legal status, political ideology, race, nationality, religion/spirituality, sex, sexual orientation, and tribal sovereign status). Efforts, as well, to protect human rights and engage in anti-racist and inclusive advocacy through sport can drive progress in society towards protecting human rights overall.

\section{Conclusion}

As is evident from the articles included in this special issue and our vision for the future, the opportunities to build partnerships across youth sport settings and the social work profession are growing and ever-evolving. We have undoubtedly only begun to scratch the surface of the ways social work and sport go hand-in-hand in tackling complex social issues. Future research and practice innovations are needed to evaluate the long-term implications of prevention and intervention programs embedded in youth sport and delivered by social workers. Other professionals (such as coaches) also may be implementing important PYD programming designed to win more than games and prepare youth for life. We also recommend continual advocacy and policy analysis to ensure equity and safety remain top priorities in youth sports. We are grateful to the editors of Child \& Adolescent Social Work Journal and all contributing authors for highlighting what we do through our social work research, practice, and advocacy efforts in sport contexts, showcasing how we contribute to social change across a variety of systems and sharing our collective impact through youth sports. We are hopeful this special issue foster more innovation related to using sport as a vehicle for social work practice and PYD, especially as the subdiscipline of sport social work continues to thrive over-time.

\section{Declarations}

Conflict of interest We have no known conflict of interest to disclose.

\section{References}

Anderson-Butcher, D. (2019). Youth sport as a vehicle for social development. Kinesiology Review, 8(3), 180-187.

Anderson-Butcher, D., Iachini, A., Riley, A., Wade-Mdivanian, R., Davis, J., \& Amorose, A. J. (2013). Exploring the impact of a summer sport-based youth development program. Evaluation and Program Planning, 37, 64-69.

CASEL (2017). What is SEL? Retrieved July 31, 2017, from http:// www.casel.org/what-is-sel/.

Coalter, F. (2010). The politics of sport-for-development: Limited focus programs and broad gauge problems? International Review for the Sociology of Sport, 45, 295-314. https://doi.org/10.1177/10126 90210366791

Domitrovich, C. E., Durlak, J. A., Staley, K. C., \& Weissberg, R. P. (2017). Social-emotional competence: An essential factor for promoting positive adjustment and reducing risk in school children. Child Development, 88(2), 408-416.

Eime, R. M., Young, J. A., Harvey, J. T., Charity, M. J., \& Payne, W. R. (2013). A systematic review of the psychological and social benefits of participation in sport for children and adolescents: informing development of a conceptual model of health through sport. International Journal of Behavioral Nutrition and Physical Activity, 10(1), 1-21.

Gould, D., \& Carson, S. (2008). Life skills development through sport: current status and future directions. International Review of Sport and Exercise Psychology, 1(1), 58-78.

Hermens, N., Super, S., Verkooijen, K. T., \& Koelen, M. A. (2017). A systematic review of life skill development through sports programs serving socially vulnerable youth. Research Quarterly for Exercise and Sport, 88, 408-424.

Holt, N. L., Neely, K. C., Slater, L. G., Camiré, M., Côté, J., FraserThomas, J., \& Tamminen, K. A. (2017). A grounded theory of positive youth development through sport based on results from a qualitative meta-study. International Review of Sport and Exercise Psychology, 10(1), 1-49.

Hulteen, R. M., Smith, J. J., Morgan, P. J., Barnett, L. M., Hallal, P. D., Colyvas, K. M., \& Lubans, D. R. (2017). Global participation in sport and leisure-time physical activities: A systematic review and metaanalysis. Preventive Medicine, 95, 14-25. 
Jensen, J., \& Hawkins, D. J. (2018). Ensure healthy development of all youth: 2018 fact sheet. Retrieved from https://grandchallenges forsocialwork.org/wp-content/uploads/2015/12/180604-GC-healt hy-dev.pdf.

Jones, G. J., Edwards, M. B., Bocarro, J. N., Bunds, K. S., \& Smith, J. W. (2016). An integrative review of sport-based youth development literature. Sport in Society, 20, 161-179. https://doi.org/10. 1080/17430437.2015.1124569

Karakaya, Y. E. (2014). The role of sports in social development: A conceptual analysis on social support program implementations and recommendations. Niğde University Journal of Physical Education and Sports Sciences, 8, 60-72.

Lawson, H. A., \& Anderson-Butcher, D. (2000). The social work of sport. In C. Simard, G. Thibault, C. Goulet, C. Pare, \& F. Bilodeau (Eds.), Sport for all and governmental policies (pp. 480-489). Quebec CA: International Olympic Committee.

Lerner, R. M., Lerner, J. V., Almerigi, J., Theokas, C., Phelps, E., \& Gestsd-ttir, S. Naudeau, S., Jelicic, H., Alberts, A. E., Ma, L., Smith, L. M., Bobek, D. L., Richman-Raphael, D., Simpson, I., Christiansen, E. D., \& von Eye, A. . (2005). Positive youth development, participation in community youth development programs, and community contributions of fifth grade adolescents: Findings from the first wave of the 4-H Study of Positive Youth Development. Journal of Early Adolescence, 25(1), 17-71.

Lower-Hoppe, L.E., Newman, T., Anderson-Butcher, D., \& Paluta, L. (in press). The influence of peers on life skill development in a sport-based positive youth development program. Journal of Sport for Development.

McDonough, M. H., Ullrich-French, S., \& McDavid, M. L. (2017). Helping kids connect: Participant and staff perspectives on facilitating social relationships in a physical activity-based positive youth development program for youth from low-income families. Sport, Exercise, and Performance Psychology, 7(1), 13-29.

Moore, M., \& Gummelt, G. (2018). Sport social work: Promoting the functioning and well-being of college and professional athletes. Cognella.

O’Connell, M. E., Boat, T., \& Warner, K. E. (2009). Committee on the prevention of mental disorders and substance abuse among children, youth, and young adults: Research advances and promising interventions. Preventing mental, emotional, and behavioral disorders among young people: Progress and possibilities. Washington (DC): National Academies Press (US); 2009. Available from: https://www.ncbi.nlm.nih.gov/books/NBK32775/ doi: $10.17226 / 12480$

Pierce, S., Kendellen, K., Camirie, M., \& Gould, D. (2016). Strategies for coaching life skills transfer. Journal of Sport Psychology in Action, 9(1), 1-10.

Reynolds, J. F. (2017). Jane Addams' forgotten legacy: recreation and sport. Journal of Issues in Intercollegiate Athletics, 11-18. College Sport Research Institute.

Riley, A., \& Anderson-Butcher, D. (2012). Participation in a summer sport-based youth development program for disadvantaged youth: Getting the parent perspective. Children and Youth Services Review, 34, 1367-1377. https://doi.org/10.1016/j.childyouth. 2012.03.008

Spruit, A., Van Vugt, E., Van Der Put, C., Van Der Stouwe, T., \& Stams, G. J. (2016). Sports participation and juvenile delinquency: A meta-analytic review. Journal of Youth and Adolescence, 45(4), 655-671.

The Aspen Institute Project Play. (2019). The state of play 2019: Trends and developments in youth sports. Retrieved from https://www. aspeninstitute.org/wp-content/uploads/2019/10/2019_SOP_Natio nal_Final.pdf.

The Aspen Institute. (2019). Calls for coaches: Coaching social and emotional skills in youth sports. Retrieved from https://www. aspeninstitute.org/wp-content/uploads/2019/02/CallsForCoaches_ Final_Web.pdf.

U.S. Department of Health and Social Services [DHHS]. (2019). The National Youth Sport Strategy. Office of the Assistant Secretary of Health.

Whitley, M. A., Massey, W. V., Camiré, M., Boutet, M., \& Borbee, A. (2019). Sport-based youth development interventions in the United States: A systematic review. BMC Public Health, 19(1), $1-20$.

Publisher's Note Springer Nature remains neutral with regard to jurisdictional claims in published maps and institutional affiliations. 